\title{
Delafloxacin: First Global Approval
}

\author{
Anthony Markham ${ }^{1}$
}

Published online: 26 July 2017

(C) Springer Nature 2018, corrected publication October/2018

\begin{abstract}
Delafloxacin (Baxdela ${ }^{\mathrm{TM}}$ ) is a fluoroquinolone antibacterial with activity against both gram-positive and gram-negative pathogens being developed by Melinta Therapeutics. The drug is being investigated or considered as a treatment for various bacterial infections and in June 2017 received approval in the USA for the treatment of acute bacterial skin and skin structure infections. This article summarizes the milestones in the development of delafloxacin leading to this first global approval for the treatment of acute bacterial skin and skin structure infections.
\end{abstract}

\section{Introduction}

Melinta Therapeutics (formerly Rib-X Pharmaceuticals) are developing delafloxacin $\left(\right.$ Baxdela $\left.^{\mathrm{TM}}\right)$, a fluoroquinolone antibacterial with activity against both grampositive and gram-negative pathogens, including methicillin-resistant Staphylococcus aureus (MRSA). Both oral and intravenous (IV) formulations of delafloxacin have been approved by the US FDA for the treatment of acute bacterial skin and skin structure infections (ABSSSI) [1]

The original version of this article was revised due to a retrospective Open Access request.

This profile has been extracted and modified from the AdisInsight database. AdisInsight tracks drug development worldwide through the entire development process, from discovery, through pre-clinical and clinical studies to market launch and beyond.

Anthony Markham

dru@adis.com

1 Springer, Private Bag 65901, Mairangi Bay, Auckland 0754, New Zealand and a study is currently underway evaluating the efficacy of the drug as treatment for community-acquired bacterial pneumonia. The recommended dose of delafloxacin in ABSSSI is $300 \mathrm{mg}$ IV or $450 \mathrm{mg}$ orally once every $12 \mathrm{~h}$ [1].

\subsection{Company Information}

In March 2017 Melinta Therapeutics entered into a development and commercialisation agreement with Menarini Group, granting Menarini exclusive rights to commercialise delafloxacin under its own brands in 68 countries including Europe, China, South Korea, Australia, and the Commonwealth of Independent States including Russia. Under the terms of the agreement Melinta will receive an upfront payment and near-term development and regulatory milestone payments, share the clinical costs for Baxdela indication expansion, as well as sales milestone and royalty payments from Menarini Group. Menarini will be responsible for submitting regulatory applications and pursuing pricing approvals for delafloxacin in countries where it has commercialisation rights [2].

In January 2015, Melinta Therapeutics and Eurofarma Laboratórios entered into an agreement for the commercialisation and distribution of delafloxacin in Brazil. Under the terms of the agreement, Eurofarma Laboratórios will acquire the rights to market and distribute the drug, pursuant to obtaining the regulatory approval for delafloxacin for the treatment of ABSSSIs. Melinta received \$US15 million as upfront cash and equity payments, and will be eligible to receive milestone payments and royalties on sales [3]. 


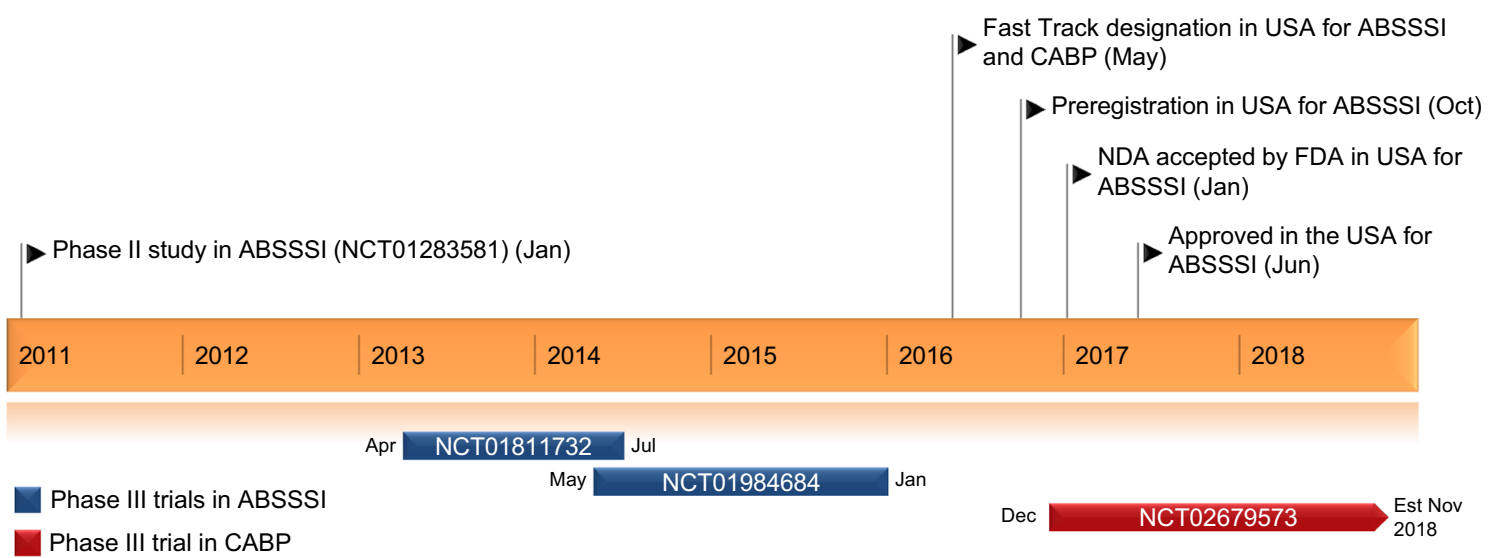

Key milestones in the development of delafloxacin. $A B S S S I$, acute bacterial skin and skin structure infections, $C A B P$ community-acquired bacterial pneumonia, Est estimated completion date

\section{Scientific Summary}

\subsection{Pharmacodynamics}

Delafloxacin had potent in vitro activity against contemporary (2014-2016) bacterial isolates frequently associated with ABSSSIs from European [4] and US [5, 6] medical centres participating in the SENTRY surveillance program. The drug had potent activity against Staphylococcus aureus ( $\mathrm{n}=903 / 3163$ European/US; $\mathrm{MIC}_{90} 0.25 \mu \mathrm{g} / \mathrm{mL}$ ) and coagulase-negative staphylococci (CoNS $[\mathrm{n}=165 / 228$ European/US] $\mathrm{MIC}_{90} \quad 0.5 \mu \mathrm{g} / \mathrm{mL}$ ) including fluoroquinolone (levofloxacin) and methicillin-resistant strains $\left(\mathrm{MIC}_{90} \leq 1 \mu \mathrm{g} / \mathrm{mL}\right)$. Delafloxacin had an $\mathrm{MIC}_{90}$ of $0.03 \mu \mathrm{g} / \mathrm{mL}$ against $\beta$-haemolytic streptococci $(\mathrm{n}=254$ / 967 European/US) and viridans group streptococci $\left(\mathrm{n}=27 / 133\right.$ European/US). The drug had an $\mathrm{MIC}_{90}$ of $1 \mu \mathrm{g} / \mathrm{mL}$ against Enterococcus faecalis $(\mathrm{n}=173 / 235),>4$ and $2 \mu \mathrm{g} / \mathrm{mL}$ against European $(\mathrm{n}=867)$ and US (1325) Enterobacteriaceae isolates, respectively, and $>4$ and $4 \mu \mathrm{g} / \mathrm{mL}$ against European $(\mathrm{n}=275)$ and US (224) Pseudomonas aeruginosa isolates, respectively. Delafloxacin had considerably greater in vitro activity than levofloxacin against E. faecalis, Enterobacteriaceae and $P$. aeruginosa (levofloxacin $\mathrm{MIC}_{90}>4 \mu \mathrm{g} / \mathrm{mL}$ ) in these studies [4-6]. Delafloxacin had an $\mathrm{MIC}_{90}$ of $0.25 \mu \mathrm{g} / \mathrm{mL}$ against $S$. aureus isolates $(\mathrm{n}=685$, including 294 methicillin-resistant $S$. aureus (MRSA) and 232 levofloxacinresistant strains) collected from patients with ABSSSIs participating in two phase III trials [7].

Delafloxacin has demonstrated bactericidal activity in vitro against MRSA [7,8] with minimum bactericidal concentrations (MBC) of $0.008,0.5$ and $8 \mu \mathrm{g} / \mathrm{mL}$ against MRSA strain 110 (levofloxacin susceptible), 124 (triple mutant) and 165 (quadruple mutant), respectively, compared to $0.5,8$ and $>32 \mu \mathrm{g} / \mathrm{ml}$ for levofloxacin. Bactericidal activity was observed at $6 \mathrm{~h}$ for most concentrations of delafloxacin and levofloxacin against MRSA strain 110; delafloxacin at 16 times MIC killed MRSA strain 124 more quickly than levofloxacin at the same concentration, and was bactericidal at 16 and 32 times MIC against MRSA strain 165 at $24 \mathrm{~h}$ [8]. In two global phase III trials, high rates of microbiological response ( $>98 \%$ eradication rates) were seen against levofloxacin nonsusceptible $S$. aureus and MRSA isolates and against isolates with mutations in the Quinolone Resistance Determining Region (QRDR) [7].<smiles>Nc1nc(-n2cc(C(=O)O)c(=O)c3cc(F)c(N4CC(O)C4)c(Cl)c32)c(F)cc1F</smiles>

Chemical structure of delafloxacin 
Delafloxacin had potent activity against $S$. aureus ( $\mathrm{n}=4$ [including $2 \mathrm{MRSA}$ strains]), Streptococcus pneumoniae ( $\mathrm{n}=4$ [including 2 penicillin-resistant strains]), and Klebsiella pneumoniae ( $\mathrm{n}=4$ [including 3 extendedspectrum $\beta$-lactamase-producing (ESBL) strains]) in vivo in a neutropenic murine lung infection model. Increasing 0.03 to $160 \mathrm{mg} / \mathrm{kg}$ doses of delafloxacin were administered once every $6 \mathrm{~h}$ to infected mice with treatment outcome measured according to the organism burden in the lung (colony forming units) after $24 \mathrm{~h}$. The median free drug area under the time-concentration curve (AUC)/MIC targets associated with net stasis were 1.42 for $S$. aureus, 0.56 for $S$. pneumoniae and 40.3 for K. pneumoniae; 1-log kill targets were 7.92, 3.36 and 55.2, respectively [9].

Delafloxacin had no clinically relevant effect on the QT/ QTc interval after administration of therapeutic (300 mg) and supratherapeutic (900 mg) IV doses in thorough QT studies in volunteers ( $\mathrm{n}=52$ and 51 , respectively) [10].

Delafloxacin showed no clinically significant phototoxic potential at all wavelengths tested after 6 days' administration of 200 or $400 \mathrm{mg} /$ day oral doses in volunteers ( $\mathrm{n}=12$ and 11 , respectively) [11].

\subsection{Pharmacokinetics}

Administration of single 300-1200 mg IV doses of delafloxacin to volunteers $(n=52)$ was associated with proportionally increasing maximum plasma concentrations $\left(\mathrm{C}_{\text {max }}\right)$ and more than proportional increases in AUC. The mean terminal half-life was $\approx 12 \mathrm{~h}$ and steady-state volume of distribution $\left(\mathrm{V}_{\mathrm{d}}\right)$ was $\approx 35$ litres. $\mathrm{C}_{\max }$ and $\mathrm{AUC}_{12}$ were $9.29 \mu \mathrm{g} / \mathrm{mL}$ and $23.4 \mu \mathrm{g} \cdot \mathrm{h} / \mathrm{mL}$, respectively, after administration of IV delafloxacin $300 \mathrm{mg}$ twice daily for 14 days to volunteers $(n=7)$. Minimal accumulation was observed (accumulation ratio 1.09) [12].

The equivalence of IV and orally administered delafloxacin has been evaluated in an open two period, two sequence crossover study in volunteers $(n=56)$. Participants were randomised to receive a single $450 \mathrm{mg}$ oral dose and a single $300 \mathrm{mg}$ IV infusion of the drug. Geometric least squares mean $\mathrm{AUC}_{\infty}$ was considered equivalent between the 2 dosage forms $(22.97$ and $26.2 \mu \mathrm{g} \cdot \mathrm{h} / \mathrm{mL}$, respectively [percent ratio 87.68 ]), however $\mathrm{C}_{\max }(5.8$ and $10.51 \mu \mathrm{g} / \mathrm{mL}$, respectively [percent ratio 55.16]) was not. These data support the use of both formulations in IV to oral switch settings in phase III clinical trials [12].

Oral administration of single 50-1600 mg doses of delafloxacin to male volunteers $(n=56)$ was associated with a dose-normalised decrease in $\mathrm{C}_{\max }$ with increasing dose; $\quad \mathrm{AUC}_{\infty}$ increased proportionally with doses $\geq 200 \mathrm{mg}$. Mean half-life was $\leq 2.5 \mathrm{~h}$ after administration of 50 and $100 \mathrm{mg}$ doses and 5.9-7.7 h with doses $\geq 200 \mathrm{mg}$. The proportion of drug excreted unchanged in urine decreased with increasing dose (from $35.6 \%$ at $50 \mathrm{mg}$ to $12.8 \%$ at $1600 \mathrm{mg}$ ) [13].

Dose-normalised $\mathrm{C}_{\max }$ and AUC decreased with increasing dose after administration of multiple oral delafloxacin 100-1200 mg once daily for 5 days to volunteers $(n=37)$. AUC values on day 5 were higher than on day 1 but accumulation was minimal (mean accumulation ratio $\leq 1.24)$. Steady state was achieved after $\approx 3$ days. Mean half-life was $4.2 \mathrm{~h}$ in the $100 \mathrm{mg} /$ day group and 7.4-8.5 $\mathrm{h}$ in the $\geq 200 \mathrm{mg} /$ day groups at day 5 [13].

Delafloxacin $\mathrm{C}_{\max }$ and $\mathrm{AUC}_{\infty}$ were significantly ( $\mathrm{p} \leq 0.001)$ higher in elderly $(\mathrm{n}=12)$ than in young volunteers $(\mathrm{n}=28)$ after administration of a single $250 \mathrm{mg}$ dose which appeared to be related to decreased creatinine clearance in the elderly cohort. The pharmacokinetic profile of the drug was similar in men and women in both the elderly and young cohorts [13].

Administration of delafloxacin $900 \mathrm{mg}$ in a formulated tablet with a high fat meal was associated with a reduction in $\mathrm{C}_{\max }$ compared with fasting conditions but this did not affect total exposure [13].

The pharmacokinetic properties of delafloxacin were similar in subjects with mild, moderate, and severe hepatic impairment (Child-Pugh class A, B, and C, respectively) compared with matched volunteers with normal hepatic function after administration of a single $300 \mathrm{mg}$ IV dose, indicating that dose adjustment of delafloxacin is not necessary in patients with hepatic impairment [14].

The mean total exposure $\left(\mathrm{AUC}_{\mathrm{t}}\right)$ of delafloxacin after a single oral $400 \mathrm{mg}$ dose was $\approx 1.5$-fold higher in patients with moderate (estimated glomerular filtration rate [eGFR] 31-50 mL/min/1.73 $\mathrm{m}^{2}$ ) and severe (eGFR $15-29 \mathrm{~mL} / \mathrm{min} / 1.73 \mathrm{~m}^{2}$ ) renal impairment compared to volunteers. However, total systemic exposures of delafloxacin in subjects with mild renal impairment (eGFR $51-80 \mathrm{~mL} / \mathrm{min} / 1.73 \mathrm{~m}^{2}$ ) was similar to that in volunteers.

The IV vehicle sulfobutylether- $\beta$-cyclodextrin (SBECD) accumulates in patients with moderate or severe renal impairment, or end stage renal disease (ESRD) on haemodialysis, with AUC increasing 2-, 5-, 7.5- and 27-fold, respectively, after administration of IV delafloxacin between $1 \mathrm{~h}$ before and $1 \mathrm{~h}$ after haemodialysis compared to volunteers. In patients with ESRD undergoing haemodialysis, SBECD is dialyzed with a clearance of $4.74 \mathrm{~L} / \mathrm{h}$. When haemodialysis occurred 1 hour after infusion of delafloxacin in patients with ESRD, the mean fraction of SBECD recovered in the dialysate was $56.1 \%$ over $\approx 4 \mathrm{~h}$ [1]. It is recommended that the dosage of IV delafloxacin be reduced to $200 \mathrm{mg}$ every $12 \mathrm{~h}$ or $200 \mathrm{mg}$ every $12 \mathrm{~h}$ with a switch to $450 \mathrm{mg}$ orally at the discretion of the physician. Administration to patients with ESRD is not recommended as there are insufficient data to recommend a dose regimen for this patient population [1]. 
Features and properties of delafloxacin

\begin{tabular}{|c|c|}
\hline Alternative names & ABT-492, Baxdela, RX-3341, RX-3341-83, WQ-3034 \\
\hline Class & Antibacterials, azetidines, fluoroquinolones, pyridines, small molecules \\
\hline $\begin{array}{l}\text { Mechanism of } \\
\text { Action }\end{array}$ & DNA gyrase inhibitors; DNA topoisomerase IV inhibitors \\
\hline $\begin{array}{l}\text { Route of } \\
\text { Administration }\end{array}$ & Oral, IV \\
\hline Pharmacodynamics & $\begin{array}{l}\mathrm{MIC}_{90} 0.25 \mu \mathrm{g} / \mathrm{mL} \text { against Staphylococcus aureus, } 0.5 \mu \mathrm{g} / \mathrm{mL} \text { against coagulase-negative staphylococci, } 0.03 \mu \mathrm{g} / \mathrm{ml} \\
\text { against } \beta \text {-haemolytic and viridans group streptococci, } 1 \mu \mathrm{g} / \mathrm{mL} \text { against Enterococcus faecalis, and }>2 \mu \mathrm{g} / \mathrm{mL} \text { against } \\
\text { Enterobacteriaceae and Pseudomonas aeruginosa }\end{array}$ \\
\hline Pharmacokinetics & $\mathrm{C}_{\max }$ 7.45/9.29 $\mu \mathrm{g} / \mathrm{mL}$, AUC $30.8 / 23.4 \mu \mathrm{g} \cdot \mathrm{h} / \mathrm{mL}, \mathrm{CL} / \mathrm{F} / \mathrm{CL} 16.8 / 13.8 \mathrm{~L} / \mathrm{h}$ at steady state (oral/IV) \\
\hline \multicolumn{2}{|l|}{ Adverse events } \\
\hline Most frequent & Nausea, diarrhoea \\
\hline Occasional & Headache, vomiting \\
\hline \multicolumn{2}{|l|}{ Rare } \\
\hline \multicolumn{2}{|l|}{ ATC codes } \\
\hline WHO ATC code & J01M-A (fluoroquinolones) \\
\hline $\begin{array}{l}\text { EphMRA ATC } \\
\text { code }\end{array}$ & J1G (fluoroquinolones) \\
\hline Chemical name & $\begin{array}{l}\text { 1-(6-amino-3,5-difluoropyridin-2-yl)-8-chloro-6-fluoro-7-(3-hydroxyazetidin-1-yl)-4-oxo-1, 4-dihydroquinoline-3- } \\
\text { carboxylic acid }\end{array}$ \\
\hline
\end{tabular}

\subsubsection{Drug Interactions}

Coadministration of midazolam and delafloxacin in volunteers was not associated with clinically relevant changes in the pharmacokinetic profile of midazolam indicating delafloxacin can be co-administered with cytochrome $\mathrm{P} 4503 \mathrm{~A}$ substrates without regard for drug-drug interactions [15].

Oral delafloxacin should be given at least $2 \mathrm{~h}$ before or $6 \mathrm{~h}$ after antacids containing aluminium or magnesium, sucralfate, metal cations such as iron, multivitamins containing iron or zinc, or with formulations containing divalent and trivalent cations as these may interfere with the absorption of delafloxacin, resulting in sub-optimal systemic concentrations [1].

\subsection{Therapeutic Trials}

\subsubsection{Phase III}

Delafloxacin had similar efficacy to vancomycin plus aztreonam as treatment for ABSSSIs in two phase III multicentre double-blind trials. In study 302 (NCT01811732) patients were randomised to IV delafloxacin $300 \mathrm{mg}$ twice daily $(\mathrm{n}=331)$ or IV vancomycin $15 \mathrm{mg} / \mathrm{kg}$ twice daily plus twice daily aztreonam $(\mathrm{n}=329)$ for 5-14 days; $19 \%$ of patients received a single dose of short-acting antibiotic prior to enrolment. Pathogens were identified at baseline in 490 patients, most frequently $S$. aureus $[1,16]$. An objective clinical response
( $\geq 20 \%$ reduction in lesion size with no further antibiotics, major procedures or death) was seen in 78.2 and $80.9 \%$ of delafloxacin and vancomycin plus aztreonam recipients, respectively, 48-72 h after completing treatment. Complete resolution of signs and symptoms (investigatorassessed cure) was seen in 81.6 and $83.3 \%$ of patients in the respective treatment groups (ITT analysis; 96.7 and $97.5 \%$ of patients in the clinically evaluable population) at follow up (day 13-15). Similar success rates were seen at late follow-up (day 21-28) [1]. Documented or presumed bacterial eradication (microbiological response) was observed in 97.8 and $98.4 \%$ of delafloxacin and vancomycin plus aztreonam recipients, respectively, including 100 and $98.5 \%$ of patients with MRSA [16].

In study 303 (NCT01984684) patients were randomised to IV delafloxacin $300 \mathrm{mg}$ twice daily for 3 days then oral delafloxacin $450 \mathrm{mg}(\mathrm{n}=423)$, or vancomycin $15 \mathrm{mg} / \mathrm{kg}$ IV plus aztreonam $(n=427)$ for $5-14$ days. Pathogens were identified at baseline in 552 patients, most frequently $S$. aureus [1, 17]. An objective clinical response (as per study 302 above) was seen in 83.7 and $80.6 \%$ of delafloxacin and vancomycin plus aztreonam recipients, respectively, 48-72 h after commencing treatment. Complete resolution of signs and symptoms (investigatorassessed cure) was seen in 87.2 and $84.8 \%$ of patients in the respective treatment groups (ITT analysis; 96.0 and $97.0 \%$ of patients in the clinically evaluable population) at follow up (day 13-15). Similar success rates were seen at late follow-up (day 21-28) [1]. Documented or presumed eradication of MRSA was observed 96 and $97 \%$ of 
delafloxacin and vancomycin plus aztreonam recipients, respectively [17].

\subsubsection{Phase II}

Delafloxacin had similar efficacy to tigecycline (study 201 [NCT0719810]) [18] as treatment for a variety of complicated skin and skin-structure infections and was similar to linezolid and superior to vancomycin as treatment for ABSSSI (study 202 [NCT01283581]) [19] in randomised double-blind phase II trials.

Study 201 compared IV treatment with delafloxacin 300 or $450 \mathrm{mg}$ once every $12 \mathrm{~h}$, or IV tigecycline $(100 \mathrm{mg}$ initial dose then $50 \mathrm{mg}$ once every $12 \mathrm{~h}$ ) for $12-14$ days in patients with various complicated skin and skin-structure infections (wound infections following surgery, trauma, burns, or animal/insect bites, abscesses, and cellulitis). Clinical cure (completely resolved or improved to the extent that additional treatment was not necessary) was achieved in 33 of 35 (94.3\%), 37 of 40 (92.5\%) and 31 of $34(91.2 \%)$ evaluable patients, in the delafloxacin 300 and $450 \mathrm{mg}$ twice daily groups and the tigecycline group, respectively, at the test-of-cure visit 14-21 days after the final dose of study drug. Infections caused by $S$. aureus (including MRSA) were effectively treated in all three groups and microbiological eradication rates were similar to clinical response by pathogen rates [18].

Study 202 compared delafloxacin $300 \mathrm{mg}$, linezolid $600 \mathrm{mg}$ and vancomycin $15 \mathrm{mg} / \mathrm{kg}$, all administered IV twice daily for 5-14 days, in patients with ABSSSIs. Investigator-assessed intention-to-treat clinical cure (complete resolution of ABSSSI signs and symptoms) at followup (day 13-15) was achieved in 57 of 81 (70.4\%) delafloxacin recipients compared to 53 of 98 (54.1) vancomycin $(\mathrm{p}<0.05)$ and 50 of $77(64.9 \%)$ linezolid recipients. The microbiological response was presumed eradicated in 88.2, 80.8 and $82.1 \%$ of microbiologically evaluable delafloxacin, vancomycin and linezolid recipients, respectively. Apart from one linezolid recipient in whom response was documented persistent, all other responses were presumed persistent [19].

\subsection{Adverse Events}

Adverse events occurring in at least $2 \%$ of delafloxacin recipients participating in the 2 phase III ABSSSI trials included nausea ( 8 and $6 \%$ of delafloxacin $[\mathrm{n}=741]$ and vancomycin plus aztreonam $[\mathrm{n}=751]$ recipients, respectively), diarrhoea ( 8 and 3\%), headache (3 and 6\%), transaminase elevations ( 3 and 4\%; pooled reports; includes hypertransaminasaemia, increased transaminases, and increased ALT and AST) and vomiting (2 and 2\%) [1]. No cases meeting the Hy's law definition have been reported with delafloxacin in clinical trials [17]. Adverse events occurring at a rate of $<2 \%$ in delafloxacin recipients included sinus tachycardia, palpitations, bradycardia, tinnitus, vertigo, blurred vision, infusion site extravasation, infusion site reactions (including bruising, discomfort, oedema, erythema, irritation, pain, phlebitis, swelling and thrombosis), abdominal pain, dyspepsia, hypersensitivity, Clostridium difficile infection, fungal infection, oral candidiasis, vulvovaginal candidiasis, hyperglycaemia, hypoglycaemia, myalgia, dizziness, hypoesthesia, paraesthesia, dysgeusia, presyncope, syncope, anxiety, insomnia, abnormal dreams, renal impairment or failure, pruritus, urticaria, dermatitis, rash, flushing, hypotension, hypertension and phlebitis [1].

Prescribing information for delafloxacin includes a boxed warning that fluoroquinolones have been associated with disabling and potentially irreversible serious adverse reactions including tendinitis and tendon rupture, peripheral and central nervous system effects, and states that delafloxacin should be discontinued in patients who experience any of these. Use of delafloxacin should also be avoided in patients with myasthenia gravis [1].

Key clinical trials of delafloxacin (Melinta Therapeutics)

\begin{tabular}{|c|c|c|c|c|c|}
\hline Drugs(s) & Indication & Phase & Status & Location(s) & Identifier \\
\hline Delafloxacin, tigecycline & Complicated skin infections & II & Completed & $\begin{array}{l}\text { US, Puerto } \\
\text { Rico }\end{array}$ & NCT00719810 \\
\hline Delafloxacin, linezolid, vancomycin & Bacterial skin infections & II & Completed & US & NCT01283581 \\
\hline Delafloxacin, ceftriaxone & Uncomplicated gonorrhoea & III & Terminated & US & NCT02015637 \\
\hline Delafloxacin, vancomycin, aztreonam & Skin and skin structure infections & III & Completed & Multinational & NCT01984684 \\
\hline $\begin{array}{l}\text { Delafloxacin, vancomycin, aztreonam, } \\
\text { placebo }\end{array}$ & Skin and skin structure infections & III & Completed & Multinational & NCT01811732 \\
\hline Delafloxacin, moxifloxacin, linezolid & $\begin{array}{l}\text { Community-acquired bacterial } \\
\text { pneumonia }\end{array}$ & III & Recruiting & Multinational & NCT02679573 \\
\hline
\end{tabular}




\subsection{Ongoing Clinical Trials}

A phase III study (DEFINE-CABP; NCT02679573) comparing delafloxacin and moxifloxacin as treatment for community-acquired bacterial pneumonia is currently recruiting patients.

\section{Current Status}

Delafloxacin received its first global approval on the 19 June 2017 in the USA for the treatment of acute bacterial skin and skin structure infections caused by susceptible bacteria.

\section{Compliance with Ethical Standards}

Funding The preparation of this review was not supported by any external funding

Conflicts of interest During the peer review process the manufacturer of the agent under review was offered an opportunity to comment on the article. Changes resulting from any comments received were made by the author on the basis of scientific completeness and accuracy. A. Markham is a contracted employee of Adis/Springer, is responsible for the article content and declares no relevant conflicts of interest. Additional information about this Adis Drug Review can be found at http://www.medengine.com/Redeem/44E8F06046B3A200.

Open Access This article is distributed under the terms of the Creative Commons Attribution-NonCommercial 4.0 International License (http://creativecommons.org/licenses/by-nc/4.0/), which permits any noncommercial use, duplication, adaptation, distribution and reproduction in any medium or format, as long as you give appropriate credit to the original author(s) and the source, provide a link to the Creative Commons license and indicate if changes were made.

\section{References}

1. Melinta Therapeutics. BAXDELA (delafloxacin): US Prescribing Information. 2017. http://www.baxdelarx.com/docs/baxdelaprescribing-information.pdf. Accessed 14 Jul 2017.

2. Melinta Therapeutics. Melinta Therapeutics and Menarini Group enter into commercial and co-development agreement for delafloxacin in 68 countries [media release] 1 Mar 2017. http://www. melinta.com.

3. Melinta Therapeutics. Melinta Therapeutics and Eurofarma Laboratorios enter into commercialization and distribution agreements for delafloxacin in Brazil [media release] 8 Jan 2015. http://www.melinta.com.

4. Huband MD, Streit JM, Shortridge D, et al. In vitro evaluation of delafloxacin activity when tested against contemporary ABSSSI isolates from Europe and surrounding areas (2014-2016): results from the SENTRY antimicrobial surveillance program [abstract no. P1351 plus poster]. In: ECCMID. 2017.
5. Shortridge D, Streit JM, Huband MD, et al. In vitro evaluation of delafloxacin activity when tested against contemporary ABSSSI isolates from the United States (2014-2016): results from the SENTRY antimicrobial surveillance Program [abstract no. Sunday-4]. In: ASM Microbe. 2017.

6. Pfaller MA, Sader HS, Rhomberg PR, et al. In vitro activity of delafloxacin against contemporary bacterial pathogens from the United States and Europe, 2014. Antimicrob Agents Chemother. 2017;61(4):e02609-16.

7. McCurdy S, Lawrence L, Quintas M, et al. In vitro activity of delafloxacin and microbiological response against fluoroquinolone susceptible and non-susceptible $S$. aureus isolates from two phase 3 studies of acute bacterial skin and skin structure infections (ABSSSI). Antimicrob Agents Chemother. 2017. doi:10.1128/AAC.00772-17.

8. Remy JM, Marra A, Duffy E. Bactericidal activity of delafloxacin against recent isolates of Staphylococcus aureus [abstract no. SUNDAY-472]. In: ASM Microbe. 2016.

9. Lepak AJ, Andes DR. In vivo pharmacodynamic target assessment of delafloxacin against Staphylococcus aureus, Streptococcus pneumoniae, and Klebsiella pneumoniae in a murine lung infection model. Antimicrob Agents Chemother. 2016;60(8): 4764-9.

10. Litwin JS, Benedict MS, Thorn MD, et al. A thorough QT study to evaluate the effects of therapeutic and supratherapeutic doses of delafloxacin on cardiac repolarization. Antimicrob Agents Chemother. 2015;59(6):3469-73.

11. Lawrence LE, Ferguson J, Paulson S. Assessment of phototoxicity potential of delafloxacin in healthy male and female subjects: a phase 1 study [abstract no. F-1198a]. In: ICAAC/ICC. 2015.

12. Hoover R, Hunt T, Benedict M, et al. Safety, tolerability, and pharmacokinetic properties of intravenous delafloxacin after single and multiple doses in healthy volunteers. Clin Ther. 2016;38(1):53-65.

13. Hoover R, Hunt $T$, Benedict $M$, et al. Single and multiple ascending-dose studies of oral delafloxacin: effects of food, sex, and age. Clin Ther. 2016;38(1):39-52.

14. Hoover R, Marbury TC, Preston RA, et al. Clinical pharmacology of delafloxacin in patients with hepatic impairment. J Clin Pharmacol. 2017;57(3):328-35.

15. Paulson SK, Wood-Horrall RN, Hoover R, et al. The pharmacokinetics of the CYP3A substrate midazolam after steady-state dosing of delafloxacin. Clin Ther. 2017;39(6):1182-90.

16. Cammarata S, Gardovskis J, Farley B, et al. Results of a global phase 3 study of delafloxacin (DLX) compared to vancomycin with aztreonam (VAN) in acute bacterial skin and skin structure infections (ABSSSI) [abstract no. 776 plus poster]. In: ICAAC/ ICC. 2015.

17. O'Riordan W, Mc Manus A, Teras J, et al. A global phase 3 study of delafloxacin compared to vancomycin/aztreonam in patients with acute bacterial skin and skin structure infections [abstract no. 1347 plus poster]. In: IDWeek. 2016.

18. O'Riordan W, Mehra $\mathrm{P}$, Manos $\mathrm{P}$, et al. A randomized phase 2 study comparing two doses of delafloxacin with tigecycline in adults with complicated skin and skin-structure infections. Int $\mathbf{J}$ Infect Dis. 2015;30:67-73.

19. Kingsley J, Mehra P, Lawrence LE, et al. A randomized, doubleblind, Phase 2 study to evaluate subjective and objective outcomes in patients with acute bacterial skin and skin structure infections treated with delafloxacin, linezolid or vancomycin. J Antimicrob Chemother. 2016;71(3):821-9. 